\title{
Article \\ Intermittent Fasting Improves High-Fat Diet-Induced Obesity Cardiomyopathy via Alleviating Lipid Deposition and Apoptosis and Decreasing m6A Methylation in the Heart
}

\author{
Zujie Xu ${ }^{1} \mathbb{D}$, Ying Qin ${ }^{1}$, Binbin Lv ${ }^{1}$, Zhenjun Tian ${ }^{2, *}$ and Bing Zhang ${ }^{1, *}$ \\ 1 Department of Physical Education, Tsinghua University, Beijing 100081, China; \\ xuzj20@mails.tsinghua.edu.cn (Z.X.); qin-y20@mails.tsinghua.edu.cn (Y.Q.); \\ lbb20@mails.tsinghua.edu.cn (B.L.) \\ 2 Institute of Sports Biology, College of Physical Education, Shaanxi Normal University, Xi'an 710119, China \\ * Correspondence: tianzhj@snnu.edu.cn (Z.T.); bzhang@mail.tsinghua.edu.cn (B.Z.)
}

\section{check for} updates

Citation: Xu, Z.; Qin, Y.; Lv, B.; Tian, Z.; Zhang, B. Intermittent Fasting Improves High-Fat Diet-Induced Obesity Cardiomyopathy via Alleviating Lipid Deposition and Apoptosis and Decreasing m6A Methylation in the Heart. Nutrients 2022, 14, 251. https://doi.org/ $10.3390 /$ nu14020251

Academic Editor: Teresa

Vanessa Fiorentino

Received: 19 December 2021

Accepted: 4 January 2022

Published: 7 January 2022

Publisher's Note: MDPI stays neutral with regard to jurisdictional claims in published maps and institutional affiliations.

Copyright: (c) 2022 by the authors. Licensee MDPI, Basel, Switzerland. This article is an open access article distributed under the terms and conditions of the Creative Commons Attribution (CC BY) license (https:// creativecommons.org/licenses/by/ $4.0 /)$.

\begin{abstract}
Intermittent fasting (IF) plays an essential role in improving lipid metabolism disorders caused by metabolic cardiomyopathy. Growing evidence revealed that N6-methyladenosine (m6A) RNA methylation is related to obesity and lipid metabolic. Our study aimed to assess the beneficial effects of IF on lipid deposition, apoptosis, and m6A methylation in high-fat diet (HFD)-induced obesity cardiomyopathy. Male C57BL/6J mice were fed a normal diet (ND) or HFD ad libitum for 13 weeks, after which time a subgroup of HFD mice were subjected to IF for $24 \mathrm{~h}$ and fed HFD in the other day for 8 weeks. We found that IF intervention significantly improved cardiac functional and structural impairment and serum lipid metabolic disorder induced by HFD. Furthermore, IF intervention decreased the mRNA levels of the fatty acid uptake genes of FABP1, FATP1, and CD36 and the fatty acid synthesis genes of SREBF1, FAS, and ACC $\alpha$ and increased the mRNA levels of the fatty acid catabolism genes of ATGL, HSL, LAL, and LPL in cardiac tissueof HFD-induced obese mice. TUNEL-positive cells, Bax/Bcl-2 ratio, and Cleaved Caspase-3 protein expression in HFD-induced obese mice hearts was down-regulated by IF intervention. In addition, IF intervention decreased the m6A methylation levels and METTL3 expression and increased FTO expression in HFD-induced obesity cardiomyopathy. In conclusion, our findings demonstrate that IF attenuated cardiac lipid deposition and apoptosis, as well as improved cardiac functional and structural impairment in HFD-induced obesity cardiomyopathy, by a mechanism associated with decreased m6A RNA methylation levels.
\end{abstract}

Keywords: intermittent fasting; high-fat diet; N6-methyladenosine methylation; obesity cardiomyopathy; lipid deposition; apoptosis

\section{Introduction}

Obesity cardiomyopathy is defined as obesity-induced impairment in the cardiac architecture and function that is independent of hypertension, coronary heart disease, and other heart diseases [1,2]. High-fat diet (HFD)-induced obesity cardiomyopathy is characterized by abnormal heart structure and dysfunction, such as echocardiographic changes consistent with poor systolic function, enhanced cardiac lipid deposition, and apoptosis [3-5]. Clinical and experimental evidence has demonstrated that myocardial lipid metabolic disorder is the initial cellular pathogenesis of obesity cardiomyopathy, which causes cardiomyocyte injury by triggering apoptosis [6-8]. Hence, further carrying out effective research on the regulation of myocardial lipid deposition is necessary and could provide new insights into potential therapeutic approaches for obesity cardiomyopathy.

Intermittent fasting (IF), a nutritional approach in which ad libitum feeding is alternated with fasting periods, has been shown to have cardioprotective effects in the heart $[9,10]$. Both clinical and experimental evidence has revealed that IF extends lifespan, decreases myocardial 
triglyceride accumulation, inhibits cardiac cell apoptosis, improves cardiac diastolic parameters, and activates a cardioprotective metabolic program [11-13]. Furthermore, growing evidence showed that IF participates in attenuating abnormal lipid metabolism [14,15]. IF ameliorates HFD or high-fructose diet-induced myocardial injury by modulating the left ventricular renin-angiotensin system [16]. In addition, IF exerts beneficial lipid metabolic effects by improving gut microbiota in HFD-induced obese mice [17]. However, the underlying mechanism by which IF regulates lipid metabolism remains unknown.

N6-methyladenosine (m6A) methylation, the most common and abundant epigenetic modification of eukaryotic mRNA, is catalyzed by m6A methyltransferase, or writers ( methyltransferase like 3/14 (METTL3/14) and Wilms tumor 1-associated protein (WTAP)), removed by m6A demethylating enzymes, or erasers ( fat mass and obesity-associated protein (FTO) and $\alpha$-ketoglutarate-dependent homolog 5 (ALKBH5)), and recognized by m6A-binding proteins, or readers (YTH domain-containing family 1/2/3 (YTHDF1/2/3) and YTH domain-containing protein 1/2 (YTHDC1/2)) [18]. Modification of m6A on mRNA functionally affects multiple RNA processes, including stability, splicing, translation, and degradation [19]. Recent studies show m6A methylation is related to obesity, lipid metabolism, and apoptosis, and plays an essential role in the physiological and pathological processes of cardiovascular diseases [20-22]. However, the effect of IF on m6A methylation modification remains unclear.

We assumed that IF in a HFD improved myocardial lipid deposition and apoptosis due to changes in m6A modification levels, thereby ameliorating obesity cardiomyopathy. Therefore, our study was designed to assess the effects of IF on lipid deposition, apoptosis, and m6A methylation in cardiac tissueof obese mice.

\section{Materials and Methods}

\subsection{Animal and Diets}

All animal experiments in our study were reviewed and approved by the Institutional Animal Care and Use Committee of Tsinghua University (identification number: F1600228; A5061-01). Three- to four-week-old male C57BL/6J mice were purchased from the Laboratory Animal Research Center of Tsinghua University and bred in the specific pathogen-free experimental animal environment at the Laboratory Animal Research Center of Tsinghua University, with five mice per cage and a $12 \mathrm{~h}$ light/12 $\mathrm{h}$ dark cycle in a temperature-controlled environment.

After 1 week of acclimation, the mice were randomly divided into 2 groups: normal diet (ND, $n=15)$ and HFD $(n=30)$. ND has $20 \% \mathrm{kcal}$ from protein, $70 \% \mathrm{kcal}$ from carbohydrates, and 10\% kcal from fat, and HFD has $20 \% \mathrm{kcal}$ from protein, $20 \% \mathrm{kcal}$ from carbohydrates, and 60\% kcal from fat (Beijing Keao Xieli Feed Co., Ltd., Beijing, China). After 13 weeks of being fed HFD, each of the 30 mice gained 20\% more body weight than at week 0 . Therefore, it can be considered that the obesity model was established. The mice fed HFD were further divided into 2 groups to continue having ad libitum access to HFD or to have IF access to food (HFD-IF, $n=15$ ) for 8 weeks. Mice in the HFD-IF group were allowed free access to regular chow every other day and no food on the alternate day [23]. No mice were excluded during the 21-week experiment. The experimental design is shown in Figure 1. The body weight and food consumption were recorded weekly. At the end of the experiment, the eyeball blood of mice was taken after fasting for $12 \mathrm{~h}$. The myocardial tissues were rapidly removed, washed with cold normal saline, dried by blotting on filter paper, weighed, and stored at $-80^{\circ} \mathrm{C}$ for later use.

\subsection{Echocardiography}

The cardiac physiological functions were assessed in anesthetized (isoflurane) mice using a two-dimensional guided M-mode echocardiography (VINNO 6 VET, VINNO, Suzhou, China) [24]. The left ventricle internal dimension diastole (LVIDd), left ventricle internal dimension systole (LVIDs), and ejection fraction (EF) were evaluated by M-mode 
echocardiography. Fractional shortening (FS) was calculated with the following formula: $\mathrm{FS}=(\mathrm{LVIDd}-$ LVIDs $) /$ LVIDd $\times 100 \%$.

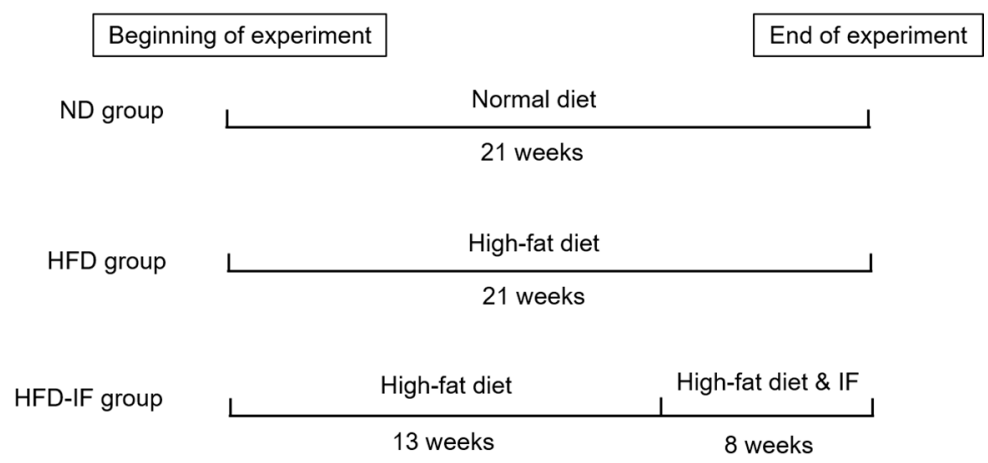

Figure 1. Schematic diagram of experimental design. Normal diet (ND) group: mice were fed ND for 21 weeks. High-fat diet (HFD) group: mice were fed HFD for 21 weeks. HFD-IF group: mice were fed HFD for 13 weeks and subjected to intermittent fasting (IF) for $24 \mathrm{~h}$ and fed HFD in the other day for 8 weeks.

\subsection{Biochemical Parameters}

Serum levels of glucose (GLU), total cholesterol (TC), triglycerides (TG), high-density lipoprotein (HDL), and low-density lipoprotein (LDL) were measured with a biochemical analyzer (KHB-ZY 1280, Shanghai Kehua Bio-engineering Co., Ltd., Shanghai, China). Free fatty acid (FFA) contents in serum were determined by an ELISA kit (ab65341, Abcam, Cambridge, MA, USA) according to the manufacturer's protocols. Levels of TG in myocardial tissue were measured using a commercially available kit (Nanjing Jiancheng Bioengineering Institute, Nanjing, China).

\section{4. m6A RNA Methylation Quantification}

An EpiQuik m6A RNA methylation quantification kit (EpiGentek, Wuhan, China) was used to quantify the global RNA m6A content in cardiac tissue samples. In brief, add $200 \mathrm{ng}$ of total RNA to the well, incubate with the capture antibody for $1 \mathrm{~h}$, then incubate with the detection antibody for $30 \mathrm{~min}$, and then incubate with the enhancer solution for $30 \mathrm{~min}$ at $-37^{\circ} \mathrm{C}$. Quantification was measured by reading the absorbance at $450 \mathrm{~nm}$ using a microplate spectrophotometer. The m6A levels in the total RNA was calculated by OD intensity.

\subsection{Histology and Oil Red O Staining}

The myocardial tissues were fixed in $4 \%$ paraformaldehyde overnight and embedded in paraffin. For the detection of morphological changes, 5- $\mu \mathrm{m}$-thick dewaxed sections were stained with hematoxylin and eosin (H\&E). The heart sections were stained with standard Masson trichrome staining to estimate interstitial fibrosis. To evaluate cardiac lipid accumulation, the frozen myocardial tissue was cut in Tissue-Tek OCT (Sakura-Finetek, Tokyo, Japan), and then the heart sections $(10 \mu \mathrm{m})$ were stained with Oil Red O (Solarbio, Beijing, China). Histopathology images were observed and acquired with an Olympus optical microscope (Olympus, Tokyo, Japan).

\subsection{Transmission Electron Microscopy (TEM)}

The mice left ventricle was cut into $1 \mathrm{~mm}^{3}$ piece and fixed with $2.5 \%$ glutaraldehyde in $0.1 \mathrm{M}$ sodium phosphate ( $\mathrm{pH} 7.4)$ for $24 \mathrm{~h}$ at $4{ }^{\circ} \mathrm{C}$. The heart tissue samples were embedded, cut, and stained with uranyl acetate and lead citrate. Lipid droplets (LDs) were observed by TEM (H-7650B, Ibaraki, Hitachi, Japan).

\subsection{TUNEL Staining}

Apoptosis was examined via using terminal deoxynucleotidyl transferase-mediated dUTP nick end-labeling (TUNEL) staining according to the manufacturer's protocols 
(Beyotime, Nanjing, China). $5 \mu \mathrm{m}$ heart sections were incubated with a TUNEL reagent mixture and incubated in dark at $37{ }^{\circ} \mathrm{C}$ for 1 hand then rinsed three times for 5 min each with PBS. The green positive particles were visualized under a Nikon fluorescence microscope (Nikon, Tokyo, Japan).

\subsection{Quantitative Real-Time PCR (RT-PCR)}

Total RNA of mouse cardiac was extracted using Trizol reagent. Reverse transcription was performed with the PrimeScript TM RT-PCR kit (TaKaRa, Tokyo, Japan). RT-PCR was detectedusing Quantitative PCR with SYBR Green PCR Master Mix (Beyotime, Nanjing, China) and CFX96 Real-Time PCR System (Bio-Rad, Hercules, CA, USA). The quantitative data were obtained with the $2^{-\Delta \Delta C t}$ method and normalized to GAPDH. RT-PCR analysis was performed for fatty acid binding protein 1 (FABP1), fatty acid transporter 1 (FATP1), cluster of differentiation 36 (CD36), sterol regulatory element-binding factor 1 (SREBF1), fatty acid synthase (FAS), acetyl- CoA carboxylase $\alpha(\mathrm{ACC} \alpha)$, adipose triglyceride lipase (ATGL), lysosomal acid lipase (LAL), hormone-sensitive lipase (HSL), lipoprotein lipase (LPL), METTL3, METTL14, WTAP, FTO, ALKBH5, YTHDF1, YTHDF2, YTHDF3, YTHDC1, YTHDC2, and GAPDH. All primers were produced by Sangon (Shanghai, China), and the primers used in this study are shown in Table 1.

Table 1. Primer Sequences Used for RT-PCR.

\begin{tabular}{|c|c|c|}
\hline \multirow{2}{*}{ Genes } & \multicolumn{2}{|c|}{ Primer Sequences } \\
\hline & Forward $\left(5^{\prime}-3^{\prime}\right)$ & Reverse $\left(5^{\prime}-3^{\prime}\right)$ \\
\hline FABP1 & CCATGACTGGGGAAAAAGTC & GCCTTTGAAAGTTGTCACCAT \\
\hline FATP1 & TGCACAGCAGGTACTACCGCAT & TGCGCAGTACCACCGTCAAC \\
\hline CD36 & ATTGGTCAAGCCAGCT & TGTAGGCTCATCCACTAC \\
\hline SREBP1c & AATCAGGACCATGCCG & СТСАAССТATGAAAATAAAGTTTGC \\
\hline FAS & GCGGGTTCGTGAAACTGATAA & CAGGTTGGCATGGTTGACAG \\
\hline $\operatorname{ACC} \alpha$ & GCCTCCGTCAGCTCAGATAC & ATGTGAAAGGCCAAACCATC \\
\hline ATGL & TGTTTCAGACGGAGAGAACG & GGAGGGGTGGAGGAATGAGG \\
\hline LAL & TGGAGGGACAAACCACTGA & AAGGGAATCGGACCACTTG \\
\hline HSL & СТTCTCССТCTCGTCTGCTG & AATGGTCCTCTGCCTCTGTC \\
\hline LPL & GATCCGAGTGAAAGCCGGAG & TTGTTTGTCCAGTGTCAGCCA \\
\hline METTL3 & CTGGGCACTTGGATTTAAGGAA & GTATCCCATCCAGTTGGTTTC \\
\hline METTL14 & CTGAGAGTGCGGATAGCATTG & GAGCAGATGTATCATAGGAAGCC \\
\hline WTAP & TAGACCCAGCGATCAACTTGT & CCTGTTTGGCTATCAGGCGTA \\
\hline FTO & TTCATGCTGGATGACCTCAATG & GCCAACTGACAGCGTTCTAAG \\
\hline ALKBH5 & GCATACGGCCTCAGGACATTA & TTCCAATCGCGGTGCATCTAA \\
\hline YTHDF1 & ACAGTTACCCCTCGATGAGTG & GGTAGTGAGATACGGGATGGGA \\
\hline YTHDF2 & GAGCAGAGACCAAAAGGTCAAG & CTGTGGGCTCAAGTAAGGTTC \\
\hline YTHDF3 & GATCAGCCTATGCCATATCTGAC & CСССTGGTTGACTAAAAACACC \\
\hline YTHDC1 & GGAAGCACCCAGTGTATAGGA & GGAAGCACCCAGTGTATAGGA \\
\hline YTHDC2 & GAAGATCGCCGTCAACATCG & GCTCTTTCCGTACTGGTCAAA \\
\hline GAPDH & GCAAGGACACTGAGCAAGA & GGATGGAAATTGTGAGGGAG \\
\hline
\end{tabular}

\subsection{Western Blot}

Total protein was isolated from heart tissue with a RIPA buffer, and the protein concentration was measured with a BCA assay kit. The protein samples were separated by SDS-polyacrylamide gels with suitable concentration and then transferred onto polyvinylidene fluoride (PVDF) membranes. The membranes were blocked with $5 \%$ bull serum albumin and then developed with diluted antibodies for Bax (14796, 1:1000, Cell Signaling Technology, Beverly, MA, USA), Bcl-2 (BS1511, 1:1000, Bioworld, Bloomington, USA), Cleaved Caspase-3 (9661, 1:1000, Cell Signaling Technology, Beverly, MA, USA), METTL3 (ab240595, 1:1000, Abcam, Cambridge, MA, USA), FTO (27226-1-AP, 1:1000, Proteintech, Wuhan, China), YTHDF1 (17479-1-AP, 1:1000, Proteintech, Wuhan, China), and GAPDH (10494-1-AP, 1:5000, Proteintech, Wuhan, China) overnight at $4{ }^{\circ} \mathrm{C}$. Subsequently, membranes were incubated with horseradish peroxidase- (HRP-) conjugated secondary antibody 
at room temperature for $90 \mathrm{~min}$. The membranes were observed on Image Systems (Bio-Rad, Hercules, CA, USA). Image Lab software was used for semiquantitative calculation.

\subsection{Statistical Analysis}

Results were shown as the mean \pm standard error of the mean (SEM) from at least three independent experiments. Statistical differences between the groups were analyzed using One-way analysis of variance (ANOVA) and post hoc least significant difference (LSD) multiple-comparison test. $p<0.05$ was considered statistically significant $\left({ }^{*} p<0.05\right.$, ** $p<0.01$ are indicated in figures).

\section{Results}

\subsection{IF Improves HFD-Induced Mice Obesity Cardiomyopathy}

Echocardiography was performed in HFD mice to determine whether IF had an improvement effect on cardiac function. Compared with mice fed with ND, 21-week HFD significantly decreased FS\% and EF\% and increased LVIDs and LVIDd. These changes caused by HFD were markedly restored in the hearts treated with IF (Figure 2A-E). HFD and HFD-IF did not significantly affect heart rate (Figure 2F). Furthermore, HFD feeding significantly caused a higher body weight, LV mass, and heart-to-body weight ratio, which were significantly decreased by IF intervention (Figure 2G-I). H\&E and Masson staining indicated that morphological abnormalities and interstitial fibrosis were observed in the myocardial tissues of obese mice; however, the above damage was remarkably reversed in HFD-IF groups (Figure 2J-L). Together, these results demonstrated that IF showed a promising approach in improved obesity cardiomyopathy induced by HFD, suggesting the protective effects on cardiomyocyte injury and dysfunction in obese mice.

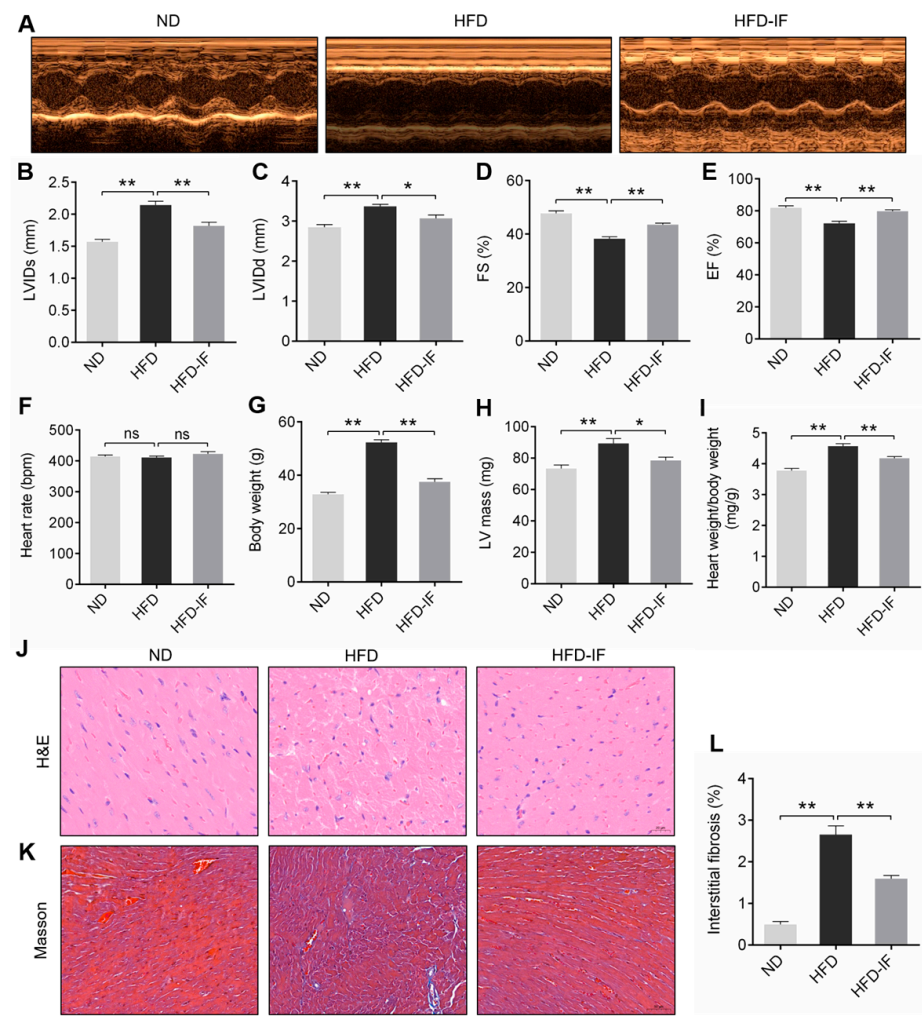

Figure 2. Effects of IF on myocardial structure and function in HFD-fed mice. (A) Echocardiographic measurements of (B) LVIDs, (C) LVIDd, (D) FS\%, and (E) EF\% for cardiac functional analysis. Measurements of (F) heart rate, $(\mathbf{G})$ body weight, $(\mathbf{H})$ LV mass, and (I) heart weight/body weight. Representative images of $(\mathbf{J}) \mathrm{H} \& \mathrm{E}$ staining (scale bar $=20 \mu \mathrm{m})$ and $(\mathbf{K})$ Masson staining (scale bar $=50 \mu \mathrm{m}$ ) cardiac sections. (L) Quantitative analysis of interstitial fibrosis. Data are shown as means \pm SEM. ${ }^{*} p<0.05,{ }^{* *} p<0.01$. ns: no significant difference. 


\subsection{IF Ameliorates HFD-Induced Serum Lipid Metabolic Disorder}

In comparison to the NC group, HFD significantly increased in serum fasting blood glucose, TC, TG, HDL, LDL, and FFA; however, these effects were remarkably mitigated in the HFD-IF groups (Figure 3A-F). It was indicated that IF could attenuate serum lipid metabolic disorder induced by HFD.

A
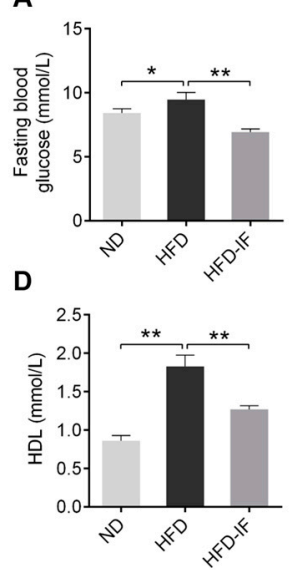

B
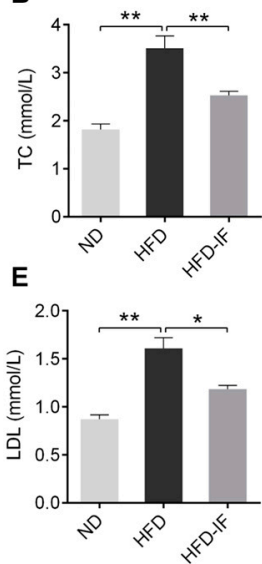

C
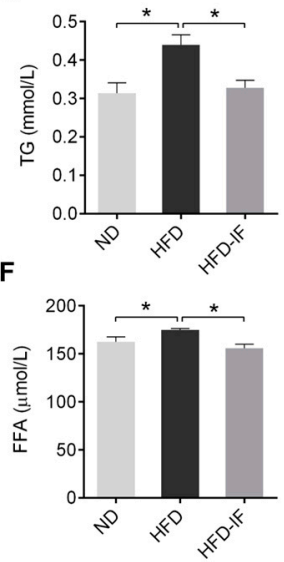

Figure 3. IF improves HFD-induced adverse lipid metabolism in serum. (A) Fasting blood glucose, (B) TC, (C) TG, (D) HDL, (E) LDL, and (F) FFA were measured in serum. Data are shown as means \pm SEM. ${ }^{*} p<0.05,{ }^{* *} p<0.01$.

\subsection{IF Alleviates HFD-Induced Cardiac Lipid Deposition}

Metabolic perturbations stemming from obesity could lead to lipid deposition in myocardium [25]. Oil Red O staining and TEM suggested that HFD significantly increased lipid deposition and LDs accumulation in hearts; these abnormalities were restored in the HFD-IF groups (Figure 4A-D). Additionally, HFD-induced lipid deposition increases the levels of TG in cardiac were down-regulated by IF intervention (Figure 4E). What's more, long-term HFD significantly increased the mRNA expression of genes regulating fatty acid uptake (FABP1, FATP1, and CD36) and fatty acid synthesis (SREBF1, FAS, and $A C C \alpha$ ), and reduced the mRNA expression of genes regulating fatty acid catabolism (ATGL, LAL, HSL, and LPL); but these effects were obviously restrained by IF intervention (Figure 4F). Collectively, these findings suggested that IF was effective in reducing cardiac lipid deposition in obese mice.

\subsection{IF Inhibites HFD-Induced Cardiac Apoptosis}

Ectopic deposition of myocardial lipids could lead to apoptosis in the heart [26,27]. TUNEL staining revealed that HFD-induced positive apoptotic particles were downregulated by IF intervention (Figure 5A,B). Consistently, western blot analysis showed that $\mathrm{Bax} / \mathrm{Bcl}-2$ ratio and Cleaved Caspase-3 protein expression were significantly increased in the HFD group compared to the ND group. However, IF significantly restored these effects (Figure 5C-E). These results demonstrated that obesity-induced apoptosis in cardiac tissues could be inhibited by IF intervention. 

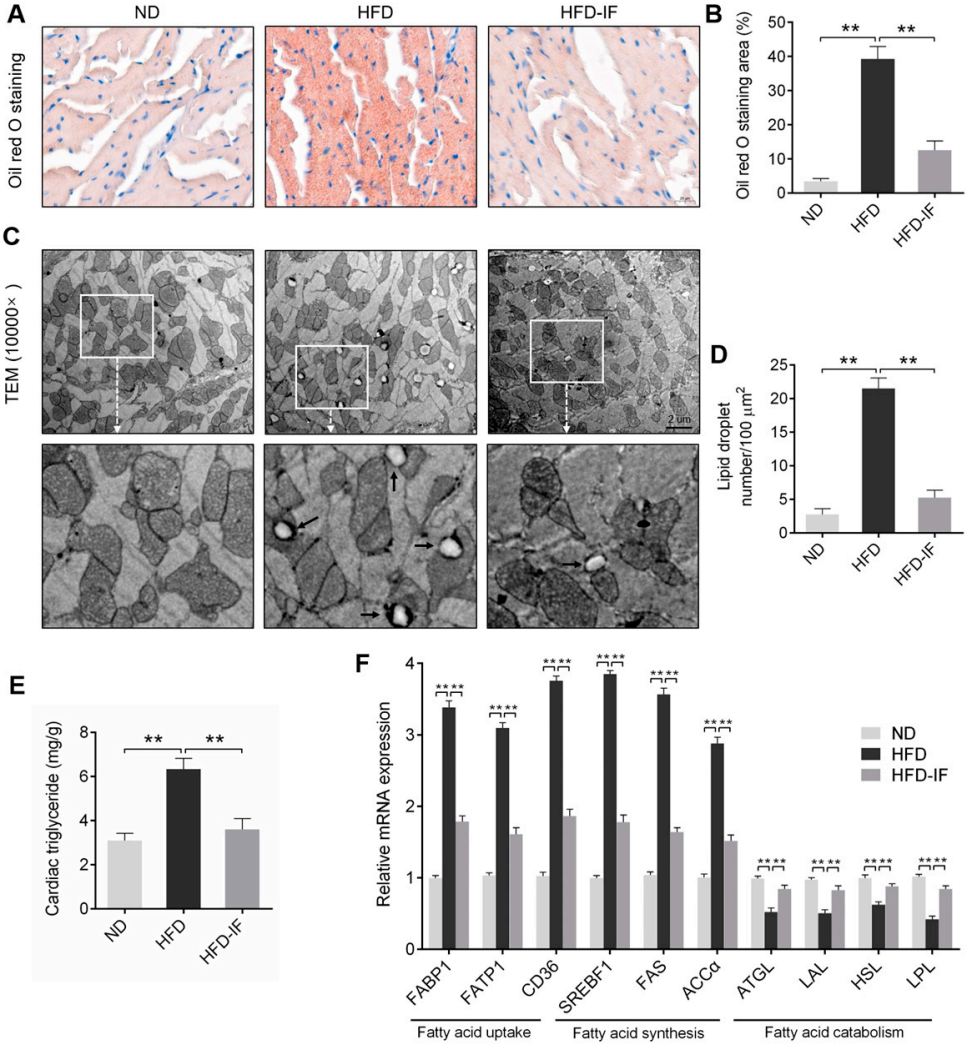

Figure 4. IF improves cardiac lipid deposition in HFD-fed mice. (A) Representative images and (B) quantitative analysis of Oil Red O staining heart sections. Scale bar $=20 \mu \mathrm{m}$. (C) LDs were observed by TEM at $10,000 \times$ magnification. Scale bar $=2 \mu \mathrm{m}$. The lower image is an amplification of the upper white box. LDs were marked with black arrows. (D) Quantitative analysis of TEM at 10,000×. (E) Levels of TG in heart of mice. (F) RT-PCR analysis of the mRNA expression of genes regulating fatty acid uptake (FABP1, FATP1, and CD36), fatty acid synthesis (SREBF1, FAS, and ACC $\alpha$ ), and fatty acid catabolism (ATGL, LAL, HSL, and LPL) in cardiac tissues. Data are shown as means \pm SEM. ${ }^{* *} p<0.01$.

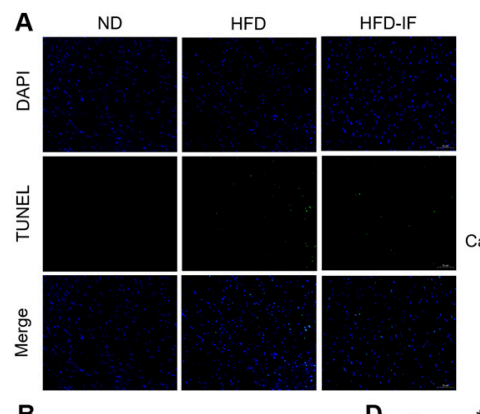

B

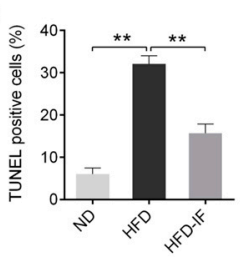

D

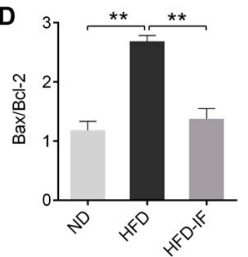

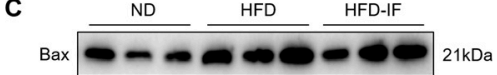

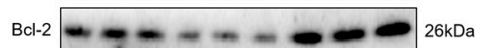

Cleaved $\longrightarrow-17 \mathrm{kDa}$

GAPDH

E

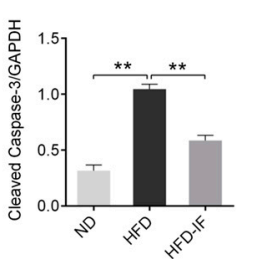

Figure 5. IF inhibits cardiac apoptosis in HFD-fed mice. (A) Representative images of TUNEL staining cardiac sections. The positive apoptotic particles in green and DAPI in blue. Scale bar $=50 \mu \mathrm{m}$. (B) Quantitative analysis of TUNEL-positive cells. (C-E) Western blot images and their densitometric quantitative analysis of Bax/Bcl-2 ratio and Cleaved Caspase-3. Data are shown as means \pm SEM. ${ }^{* *} p<0.01$. 


\subsection{IF Decreases HFD-Induced Cardiac m6A Methylation}

To assess the effect of IF on m6A methylation, we measured m6A content and m6Aassociated genes and proteins in cardiac tissues. IF intervention markedly rescued m6A content in the heart from HFD-fed mice (Figure 6A). Additionally, the gene expression of METTL3 was up-regulated by HFD, and the FTO mRNA levels was down-regulated; however, IF intervention markedly reversed HFD-induced these effects, which were also confirmed by western blot (Figure 6B-D). Taken together, these results indicated that IF intervention decreases cardiac m6A methylation levels in HFD-fed mice.

A

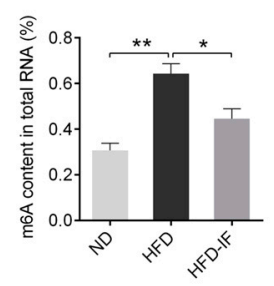

B

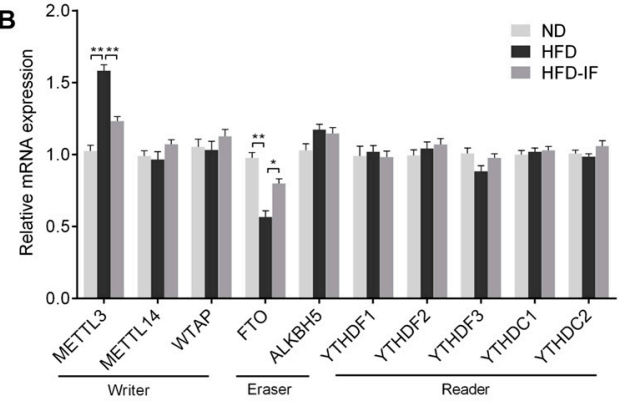

C

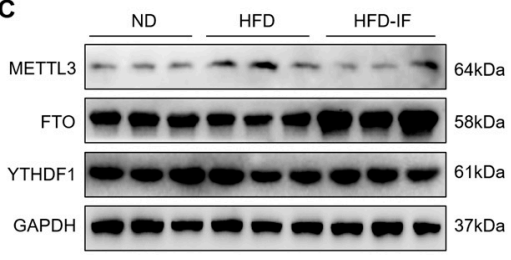

D

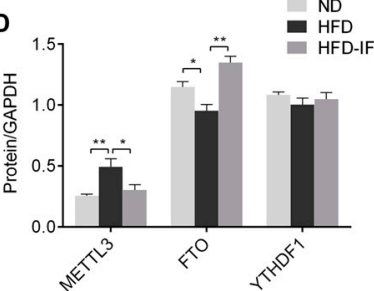

Figure 6. IF decreases cardiac m6A methylation in HFD-fed mice. (A) The m6A level in heart tissue samples. (B) RT-PCR analysis of the mRNA levels of m6A methylation-related genes in cardiac tissues related to writer (METTL3, METTL14, and WTAP), eraser (FTO and ALKBH5), and reader (YTHDF1, YTHDF2, YTHDF3, YTHDC1, and YTHDC2). (C,D) Western blot images and their densitometric quantitative analysis of METTL3, FTO, and YTHDF1. Data are shown as means \pm SEM. ${ }^{*} p<0.05$, ** $p<0.01$.

\section{Discussion}

As we know, cardiac tissueis the major attacking target of obesity. HFD-induced obesity cardiomyopathy is characterized by abnormal heart structure and dysfunction [2,3]. Increasing evidence suggests the protective effect of IF in obesity cardiomyopathy [28-30]; however, its molecular mechanism is not yet fully clarified. In the present study, we examined the therapeutic effect of IF on obesity cardiomyopathy as well as its impact on lipid deposition and m6A methylation in the heartof obese mice. Our study revealed that IF intervention protected against HFD-induced lipid deposition, apoptosis, and m6A methylation in cardiac tissues, as a result, restored cardiac functional and structural impairment. Mechanistically, IF may regulate myocardial lipid metabolism and apoptosis through m6A methylation in obesity cardiomyopathy. The present study, in part, shows that the protective effects of IF in cardiovascular protection may be involved in m6A RNA methylation.

Long-term HFD induces obese cardiomyopathy, leading to cardiac abnormal structure and dysfunction. Our results indicated that 8 weeks of IF intervention in obese mice could attenuate cardiac diastolic and systolic dysfunction, morphological abnormalities, and interstitial fibrosis. It was reported that the disorder of myocardial lipid metabolism is considered to be the major pathogenesis of obesity cardiomyopathy [3]. Previous studies have shown that serum lipid metabolism disorders in obese mice, such as increased levels of TC, TG, HDL, LDL, and FFA [31,32]. Furthermore, 24-week HFD resulted in increased cardiac TG content and LDs number and induced lipotoxic cardiomyopathy [33]. Similarly, in our results, abnormal serum lipid metabolism and cardiac lipid deposition were examined in obese mice but were ameliorated by IF intervention. Lipid synthesis and breakdown are 
highly regulated multistep reactions, with a pathway involving distinct sets of enzymatic reactions. Obesity induces the breakdown of the dynamic balance of myocardial lipid synthesis and decomposition and the abnormal transcription of the cardiac lipid accumulation, lipid synthesis, and catabolism genes [34]. We found that IF decreased the mRNA levels of fatty acid uptake genes of FABP1, FATP1, and CD36, the fatty acid synthesis genes of SREBF1, FAS, and ACC $\alpha$, and increased the gene expression of fatty acid catabolism genes of ATGL, HSL, LAL, and LPL. Therefore, we speculated that IF alleviates HFD-induced cardiac lipid deposition by decreasing fatty acid uptake and synthesis and increasing fatty acid catabolism.

Myocardial lipid accumulation has been reported to promote myocardial apoptosis [35]. Previous research has shown that myocardial apoptosis increased in obese rats, as evidenced by an up-regulated TUNEL-positive cells, Bax/Bcl-2 ratio, and Cleaved Caspase-3 protein expression [36]. Another study reported that food restriction decreased TUNEL-positive cells, Bax/Bcl-2 ratio, and Cleaved Caspase-3 protein expression, which could inhibit cardiac apoptosis in HFD-induced obese rats [28]. Similarly, in our study, TUNEL staining and western blot analysis showed that cardiac lipid deposition significantly increased cardiac apoptotic cell death, and IF intervention markedly reversed HFD-induced these effects. In sum, IF appeared to inhibit HDF-induced cardiac apoptosis, but its underlying mechanism at the epitranscriptomic modification is not fully understood yet.

Growing evidence revealed that m6A methylation is related to obesity and lipid metabolic [37,38]. Previous study has shown that the mRNA and protein expression of FTO were upregulated in non-alcoholic fatty liver disease (NAFLD) rats, which were involved in lipid metabolism disorders [39]. The m6A methylation levels and METTL3 expression were raised in the livers of obese mice, and hepatocyte-specific knockout of METTL3 improved lipid metabolic disorders and insulin resistance [40]. A recent study showed that YTHDC2, an $\mathrm{m} 6 \mathrm{~A}$ reader, was significantly down-regulated in the livers of obese mice and NAFLD patients. Overexpression of YTHDC2 in the livers of obese mice improved hepatic lipid metabolism and insulin resistance [41]. In addition, increased m6A methylation levels were up-regulated in the impaired cardiovascular system and were involved in the pathogenesis of cardiovascular diseases, leading to cardiac apoptosis and dysfunction [20]. A recent study has revealed that m6A methylated levels were up-regulated and the gene and protein levels of FTO were reduced in human and mouse failing hearts, while FTO overexpression significantly decreased fibrosis and enhanced cardiomyocyte contractile function [42]. The m6A levels and METTL3 expression were up-regulated in hypoxia/reoxygenation (H/R)treated cultured H9C2 cells and ischemia/reperfusion (I/R) mouse hearts. Cardiomyocytespecific knockout of METTL3 promoted autophagic flux and reduced apoptosis in I/R mice [43]. Our study revealed that m6A methylation levels and METTL3 were consistently up-regulated, and FTO was consistently reduced in cardiac tissueof HFD-fed mice. It is worth noting that IF intervention markedly reversed HFD-induced these effects. We speculate that down-regulation the expression of METTL3 and up-regulation the expression of FTO may be responsible for altered m6A levels in the HFD-IF group. Additionally, it has been reported that IF can improve metabolic diseases such as obesity and aging by regulating epigenetic modifications [44,45]. Therefore, it is thought that IF may ameliorate HFD-induced obesity cardiomyopathy via decreasing m6A RNA methylation.

As indicated above, to the best of our knowledge, our study is the first to assess the effects of IF on m6A RNA methylation. Together, these results provide a novel pathological mechanism of HFD-induced obesity cardiomyopathy and suggest that reducing m6A methylation levels through intermittent fasting intervention is a therapeutic strategy for obesity-associated myocardial lipid deposition and apoptosis. Our study may help to design better non-pharmacological intervention protocols for obesity cardiomyopathy patients. However, one limitation of our manuscript is that it only detected the expression of m6A-associated genes and proteins by RT-PCR and western blot but did not conduct a genome-wide profiling of m6A-tagged transcripts in cardiac tissueby methylated RNA immunoprecipitation sequencing (MeRIP-Seq). Moreover, the molecular mechanism by 
which IF can attenuate cardiac lipid deposition and apoptosis via m6A methylation in obesity cardiomyopathy will need to be explored in further study.

\section{Conclusions}

In summary, our results demonstrate that IF attenuated cardiac lipid deposition and apoptosis, as well as improved cardiac functional and structural impairment in HFDinduced obesity cardiomyopathy, by a mechanism associated with decreased m6A RNA methylation levels (Figure 7).

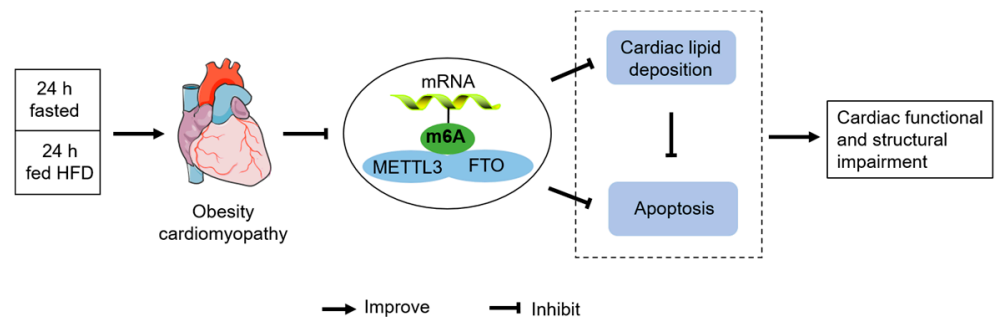

Figure 7. A possible mechanism for IF to improve obesity cardiomyopathy induced by HFD.

Author Contributions: Z.X., Z.T. and B.Z. researched and designed. Z.X., Y.Q. and B.L. conducted the experiments and analyzed the data. Z.X. wrote the manuscript. Z.T. and B.Z. edited the draft manuscript. All authors have read and agreed to the published version of the manuscript.

Funding: This work was supported by the Ministry of Education of the People's Republic of China (No. 20194180050).

Institutional Review Board Statement: All animal experiments in this study were reviewed and approved by the Institutional Animal Care and Use Committee of Tsinghua University (identification number: F16-00228; A5061-01).

Informed Consent Statement: Not applicable.

Data Availability Statement: The data presented in this study are available on request from the corresponding author.

Conflicts of Interest: The authors declare no conflict of interest.

\section{References}

1. Ren, J.; Wu, N.N.; Wang, S.; Sowers, J.R.; Zhang, Y. Obesity cardiomyopathy: Evidence, mechanisms, and therapeutic implications. Physiol. Rev. 2021, 101, 1745-1807. [CrossRef]

2. Wong, C.; Marwick, T.H. Obesity cardiomyopathy: Pathogenesis and pathophysiology. Nat. Clin. Pract. Cardiovasc. Med. 2007, 4, 436-443. [CrossRef]

3. Gutierrez-Cuevas, J.; Sandoval-Rodriguez, A.; Meza-Rios, A.; Monroy-Ramirez, H.C.; Galicia-Moreno, M.; Garcia-Banuelos, J.; Santos, A.; Armendariz-Borunda, J. Molecular mechanisms of obesity-linked cardiac dysfunction: An up-date on current knowledge. Cells 2021, 10, 629. [CrossRef]

4. Shao, D.; Kolwicz, S.C., Jr.; Wang, P.; Roe, N.D.; Villet, O.; Nishi, K.; Hsu, Y.A.; Flint, G.V.; Caudal, A.; Wang, W.; et al. Increasing fatty acid oxidation prevents high-fat diet-induced cardiomyopathy through regulating Parkin-mediated mitophagy. Circulation 2020, 142, 983-997. [CrossRef]

5. Alpert, M.A.; Omran, J.; Bostick, B.P. Effects of obesity on cardiovascular hemodynamics, cardiac morphology, and ventricular function. Curr. Obes. Rep. 2016, 5, 424-434. [CrossRef] [PubMed]

6. Sletten, A.C.; Peterson, L.R.; Schaffer, J.E. Manifestations and mechanisms of myocardial lipotoxicity in obesity. J. Intern. Med. 2018, 284, 478-491. [CrossRef]

7. Goldberg, I.J.; Trent, C.M.; Schulze, P.C. Lipid metabolism and toxicity in the heart. Cell Metab. 2012, 15, 805-812. [CrossRef]

8. Zhang, Y.; Ren, J. Role of cardiac steatosis and lipotoxicity in obesity cardiomyopathy. Hypertension 2011, 57, 148-150. [CrossRef]

9. Dong, T.A.; Sandesara, P.B.; Dhindsa, D.S.; Mehta, A.; Arneson, L.C.; Dollar, A.L.; Taub, P.R.; Sperling, L.S. Intermittent fasting: A heart healthy dietary pattern? Am. J. Med. 2020, 133, 901-907. [CrossRef]

10. Tinsley, G.M.; Horne, B.D. Intermittent fasting and cardiovascular disease: Current evidence and unresolved questions. Future Cardiol. 2018, 14, 47-54. [CrossRef]

11. Malinowski, B.; Zalewska, K.; Wesierska, A.; Sokolowska, M.M.; Socha, M.; Liczner, G.; Pawlak-Osinska, K.; Wicinski, M. Intermittent fasting in cardiovascular disorders-an overview. Nutrients 2019, 11, 673. [CrossRef] 
12. Godar, R.J.; Ma, X.; Liu, H.; Murphy, J.T.; Weinheimer, C.J.; Kovacs, A.; Crosby, S.D.; Saftig, P.; Diwan, A. Repetitive stimulation of autophagy-lysosome machinery by intermittent fasting preconditions the myocardium to ischemia-reperfusion injury. Autophagy 2015, 11, 1537-1560. [CrossRef]

13. Ahmet, I.; Wan, R.; Mattson, M.P.; Lakatta, E.G.; Talan, M. Cardioprotection by intermittent fasting in rats. Circulation 2005, 112, 3115-3121. [CrossRef]

14. Antoni, R.; Johnston, K.L.; Collins, A.L.; Robertson, M.D. Effects of intermittent fasting on glucose and lipid metabolism. Proc. Nutr. Soc. 2017, 76, 361-368. [CrossRef]

15. Wilson, R.A.; Deasy, W.; Stathis, C.G.; Hayes, A.; Cooke, M.B. Intermittent fasting with or without exercise prevents weight gain and improves lipids in diet-induced obese mice. Nutrients 2018, 10, 346. [CrossRef]

16. Camelo, L.; Marinho, T.S.; Aguila, M.B.; Souza-Mello, V.; Barbosa-da-Silva, S. Intermittent fasting exerts beneficial metabolic effects on blood pressure and cardiac structure by modulating local renin-angiotensin system in the heart of mice fed high-fat or high-fructose diets. Nutr. Res. 2019, 63, 51-62. [CrossRef] [PubMed]

17. Deng, Y.; Liu, W.; Wang, J.; Yu, J.; Yang, L.Q. Intermittent fasting improves lipid metabolism through changes in gut microbiota in diet-induced obese mice. Med. Sci. Monit. 2020, 26, e926789. [CrossRef]

18. Zaccara, S.; Ries, R.J.; Jaffrey, S.R. Reading, writing and erasing mRNA methylation. Nat. Rev. Mol. Cell Biol. 2019, 20, 608-624 [CrossRef]

19. Yang, C.; Hu, Y.; Zhou, B.; Bao, Y.; Li, Z.; Gong, C.; Yang, H.; Wang, S.; Xiao, Y. The role of m(6)A modification in physiology and disease. Cell Death Dis. 2020, 11,960. [CrossRef]

20. Wu, S.; Zhang, S.; Wu, X.; Zhou, X. m(6)A RNA methylation in cardiovascular diseases. Mol. Ther. 2020, 28, 2111-2119. [CrossRef]

21. Zhang, B.; Jiang, H.; Dong, Z.; Sun, A.; Ge, J. The critical roles of m6A modification in metabolic abnormality and cardiovascular diseases. Genes Dis. 2021, 8, 746-758. [CrossRef]

22. Kumari, R.; Ranjan, P.; Suleiman, Z.G.; Goswami, S.K.; Li, J.; Prasad, R.; Verma, S.K. mRNA modifications in cardiovascular biology and disease: With a focus on m6A modification. Cardiovasc. Res. 2021; in press. [CrossRef]

23. Li, G.; Xie, C.; Lu, S.; Nichols, R.G.; Tian, Y.; Li, L.; Patel, D.; Ma, Y.; Brocker, C.N.; Yan, T.; et al. Intermittent fasting promotes white adipose browning and decreases obesity by shaping the gut microbiota. Cell Metab. 2017, 26, 672-685. [CrossRef]

24. Wu, F.; Li, Z.; Cai, M.; Xi, Y.; Xu, Z.; Zhang, Z.; Li, H.; Zhu, W.; Tian, Z. Aerobic exercise alleviates oxidative stress-induced apoptosis in kidneys of myocardial infarction mice by inhibiting ALCAT1 and activating FNDC5/Irisin signaling pathway. Free Radic. Biol. Med. 2020, 158, 171-180. [CrossRef]

25. Battiprolu, P.K.; Hojayev, B.; Jiang, N.; Wang, Z.V.; Luo, X.; Iglewski, M.; Shelton, J.M.; Gerard, R.D.; Rothermel, B.A.; Gillette, T.G.; et al. Metabolic stress-induced activation of FoxO1 triggers diabetic cardiomyopathy in mice. J. Clin. Investig. 2012, 122, 1109-1118. [CrossRef]

26. Hsu, H.C.; Chen, C.Y.; Lee, B.C.; Chen, M.F. High-fat diet induces cardiomyocyte apoptosis via the inhibition of autophagy. Eur. J. Nutr. 2016, 55, 2245-2254. [CrossRef]

27. Zuo, A.; Zhao, X.; Li, T.; Li, J.; Lei, S.; Chen, J.; Xu, D.; Song, C.; Liu, T.; Li, C.; et al. CTRP9 knockout exaggerates lipotoxicity in cardiac myocytes and high-fat diet-induced cardiac hypertrophy through inhibiting the LKB1/AMPK pathway. J. Cell Mol. Med. 2020, 24, 2635-2647. [CrossRef]

28. Lin, Y.Y.; Hsieh, P.S.; Cheng, Y.J.; Cheng, S.M.; Chen, C.J.; Huang, C.Y.; Kuo, C.H.; Kao, C.L.; Shyu, W.C.; Lee, S.D. Anti-apoptotic and pro-survival effects of food restriction on high-fat diet-induced obese hearts. Cardiovasc. Toxicol. 2017, 17, 163-174. [CrossRef]

29. Klempel, M.C.; Kroeger, C.M.; Varady, K.A. Alternate day fasting (ADF) with a high-fat diet produces similar weight loss and cardio-protection as ADF with a low-fat diet. Metabolism 2013, 62, 137-143. [CrossRef]

30. Varady, K.A.; Hudak, C.S.; Hellerstein, M.K. Modified alternate-day fasting and cardioprotection: Relation to adipose tissue dynamics and dietary fat intake. Metabolism 2009, 58, 803-811. [CrossRef]

31. Ge, C.X.; Xu, M.X.; Qin, Y.T.; Gu, T.T.; Lou, D.S.; Li, Q.; Hu, L.F.; Wang, B.C.; Tan, J. Endoplasmic reticulum stress-induced iRhom2 up-regulation promotes macrophage-regulated cardiac inflammation and lipid deposition in high fat diet (HFD)-challenged mice: Intervention of fisetin and metformin. Free Radic. Biol. Med. 2019, 141, 67-83. [CrossRef]

32. Wu, J.; Li, Y.; Yu, J.; Gan, Z.; Wei, W.; Wang, C.; Zhang, L.; Wang, T.; Zhong, X. Resveratrol attenuates high-fat diet induced hepatic lipid homeostasis disorder and decreases m(6)A RNA methylation. Front. Pharmacol. 2020, 11, 568006. [CrossRef]

33. Wang, L.; Zhao, D.; Tang, L.; Li, H.; Liu, Z.; Gao, J.; Edin, M.L.; Zhang, H.; Zhang, K.; Chen, J.; et al. Soluble epoxide hydrolase deficiency attenuates lipotoxic cardiomyopathy via upregulation of AMPK-mTORC mediated autophagy. J. Mol. Cell Cardiol. 2021, 154, 80-91. [CrossRef]

34. Costantino, S.; Akhmedov, A.; Melina, G.; Mohammed, S.A.; Othman, A.; Ambrosini, S.; Wijnen, W.J.; Sada, L.; Ciavarella, G.M.; Liberale, L.; et al. Obesity-induced activation of JunD promotes myocardial lipid accumulation and metabolic cardiomyopathy. Eur Heart J. 2019, 40, 997-1008. [CrossRef]

35. Feng, W.; Lei, T.; Wang, Y.; Feng, R.; Yuan, J.; Shen, X.; Wu, Y.; Gao, J.; Ding, W.; Lu, Z. GCN2 deficiency ameliorates cardiac dysfunction in diabetic mice by reducing lipotoxicity and oxidative stress. Free Radic. Biol. Med. 2019, 130, 128-139. [CrossRef]

36. Lee, S.D.; Shyu, W.C.; Cheng, I.S.; Kuo, C.H.; Chan, Y.S.; Lin, Y.M.; Tasi, C.Y.; Tsai, C.H.; Ho, T.J.; Huang, C.Y. Effects of exercise training on cardiac apoptosis in obese rats. Nutr. Metab. Cardiovasc. Dis. 2013, 23, 566-573. [CrossRef]

37. Wu, J.; Frazier, K.; Zhang, J.; Gan, Z.; Wang, T.; Zhong, X. Emerging role of m(6) A RNA methylation in nutritional physiology and metabolism. Obes. Rev. 2020, 21, e12942. [CrossRef] 
38. Sun, M.; Zhang, X. Epigenetic regulation of N6-methyladenosine modifications in obesity. J. Diabetes Investig. 2021, 12, 1306-1315. [CrossRef]

39. Guo, J.; Ren, W.; Li, A.; Ding, Y.; Guo, W.; Su, D.; Hu, C.; Xu, K.; Chen, H.; Xu, X.; et al. Fat mass and obesity-associated gene enhances oxidative stress and lipogenesis in nonalcoholic fatty liver disease. Dig. Dis. Sci. 2013, 58, 1004-1009. [CrossRef]

40. Xie, W.; Ma, L.L.; Xu, Y.Q.; Wang, B.H.; Li, S.M. METTL3 inhibits hepatic insulin sensitivity via N6-methyladenosine modification of Fasn mRNA and promoting fatty acid metabolism. Biochem. Biophys. Res. Commun. 2019, 518, 120-126. [CrossRef] [PubMed]

41. Zhou, B.; Liu, C.; Xu, L.; Yuan, Y.; Zhao, J.; Zhao, W.; Chen, Y.; Qiu, J.; Meng, M.; Zheng, Y.; et al. N(6)-methyladenosine reader protein YT521-B homology domain-containing 2 suppresses liver steatosis by regulation of mRNA stability of lipogenic genes. Hepatology 2021, 73, 91-103. [CrossRef]

42. Mathiyalagan, P.; Adamiak, M.; Mayourian, J.; Sassi, Y.; Liang, Y.; Agarwal, N.; Jha, D.; Zhang, S.; Kohlbrenner, E.; Chepurko, E.; et al. FTO-dependent N(6)-methyladenosine regulates cardiac function during remodeling and repair. Circulation 2019, 139, 518-532. [CrossRef] [PubMed]

43. Song, H.; Feng, X.; Zhang, H.; Luo, Y.; Huang, J.; Lin, M.; Jin, J.; Ding, X.; Wu, S.; Huang, H.; et al. METTL3 and ALKBH5 oppositely regulate m(6)A modification of TFEB mRNA, which dictates the fate of hypoxia/reoxygenation-treated cardiomyocytes. Autophagy 2019, 15, 1419-1437. [CrossRef]

44. Asif, S.; Morrow, N.M.; Mulvihill, E.E.; Kim, K.H. Understanding dietary intervention-mediated epigenetic modifications in metabolic diseases. Front. Genet. 2020, 11, 590369. [CrossRef] [PubMed]

45. Gensous, N.; Franceschi, C.; Santoro, A.; Milazzo, M.; Garagnani, P.; Bacalini, M.G. The impact of caloric restriction on the epigenetic signatures of aging. Int. J. Mol. Sci. 2019, 20, 2022. [CrossRef] 